\title{
Team-optimal online estimation of dynamic parameters over distributed tree networks
}

\author{
O. Fatih Kilic ${ }^{\mathrm{a}, *}$, Tolga Ergen ${ }^{\mathrm{b}}$, Muhammed O. Sayin ${ }^{\mathrm{c}}$, Suleyman S. Kozat ${ }^{\mathrm{b}}$ \\ ${ }^{a}$ Department of Electrical and Computer Engineering, University of Texas at Austin, Austin, TX 78705, USA \\ ${ }^{\mathrm{b}}$ Department of Electrical and Electronics Engineering, Bilkent University, Ankara, Turkey \\ ${ }^{\mathrm{c}}$ Department of Electrical and Computer Engineering, University of Illinois at Urbana-Champaign, Champaign, IL 61801, USA
}

\section{A R T I C L E I N F O}

\section{Article history:}

Received 19 December 2017

Revised 24 May 2018

Accepted 11 August 2018

Available online 22 August 2018

\section{Keywords:}

Optimal estimation

Distributed network

Tree networks

Dynamic parameter

Online estimation

\begin{abstract}
A B S T R A C T
We study online parameter estimation over a distributed network, where the nodes in the network collaboratively estimate a dynamically evolving parameter using noisy observations. The nodes in the network are equipped with processing and communication capabilities and can share their observations or local estimates with their neighbors. The conventional distributed estimation algorithms cannot perform the team-optimal online estimation in the finite horizon global mean-square error sense (MSE). To this end, we present a team-optimal distributed estimation algorithm through the disclosure of local estimates for tracking an underlying dynamic parameter. We first show that the optimal estimation can be achieved through the diffusion of all the time stamped observations for any arbitrary network and prove that the team optimality through disclosure of local estimates is only possible for certain network topologies such as tree networks. We then derive an iterative algorithm to recursively calculate the combination weights of the disclosed information and construct the team-optimal estimate for each time step. Through series of simulations, we demonstrate the superior performance of the proposed algorithm with respect to the state-of-the-art diffusion distributed estimation algorithms regarding the convergence rate and the finite horizon MSE levels. We also show that while conventional distributed estimation schemes cannot track highly dynamic parameters, through optimal weight and estimate construction, the proposed algorithm presents a stable MSE performance.
\end{abstract}

(c) 2018 Elsevier B.V. All rights reserved.

\section{Introduction}

\subsection{Preliminaries}

Recently, due to advancements in information technologies, distributed learning and estimation techniques have attracted significant attention thanks to their fast convergence and robustness properties for fast streaming data [1-7]. In a distributed estimation framework, we consider a network of agents observing a temporal signal about an underlying state, possibly coming from different spatial sources with different statistics. Each agent in the network is equipped with communication and processing capabilities. The aim of each agent is to estimate the underlying parameter of interest, as an example, by minimizing the expected Euclidean distance between the estimate and the true value of the

\footnotetext{
* Corresponding author.

E-mail addresses: okilic@utexas.edu (O.F. Kilic),ergen@ee.bilkent.edu.tr (T. Ergen), sayin2@illinois.edu (M.O. Sayin), kozat@ee.bilkent.edu.tr (S.S. Kozat).
}

state (the minimum mean-square estimation (MMSE)). The agents in the network are connected to a set of neighboring nodes and can exchange information, i.e. observations and/or estimates, between them to improve their learning process. To illustrate, assume a network of emission sensors distributed over a greenhouse to monitor the $\mathrm{CO}_{2}$ levels for a precision agriculture application [8]. Since the agents would collect different observations from different parts of the area, they can cooperate in the network to rapidly learn and track the true $\mathrm{CO}_{2}$ levels for an enhanced intervention.

In this regard, the distributed learning and estimation has been extensively studied in the signal processing and machine learning literatures [9-17]. However, the classical methods either do not consider the information diffusion scheme among the agents and/or construction of the optimal combination methods to obtain the MMSE performance or are not applicable for real-time applications [13]. To this end, in this paper, we present an approach to obtain the optimal distributed online estimation in a team framework by exploiting the network structure and the information disclosure and combination when the underlying state is non-stationary and time varying. In this framework, agents in our network cooperate 
with their neighbors as a "team" to minimize a predefined team cost based on the actions of each agent. To achieve this aim, each agent generates a local estimate for the underlying dynamic system parameter and then constructs certain messages to share with its neighbors at each time instance. Based on the sharing process, each agent's goal is to obtain a solution that minimizes the predefined team cost, i.e., the "team-optimal" solution.

\subsection{Related work}

There exists an extensive research on distributed estimation of a time invariant or a dynamic state parameter, which are mainly studied under centralized and decentralized distributed learning frameworks $[9-16,18,19]$. In the centralized frameworks, all the agents in the network are connected to a fusion center and each agent transmits its information to the center for the construction of the final estimate $[9,18,19]$. Since all the information is collected by a single node such methods do not require any specific information sharing scheme and constructing the global optimal estimate is straightforward. However, this approach has serious disadvantages regarding communication and computation loads on the network, i.e. transmitting all the peripheral information to a single node requires a huge communication bandwidth and processing all the collected information on a single unit requires a significant computational power $[9,12]$.

In the alternative decentralized frameworks, each agent in the network has a different set of neighboring nodes consisting of spatially close ones and exchange information only with these nodes to overcome the former problems [20]. In these approaches, agents only disclose (or share) their local information on the underlying parameter and combine the received information to produce their final estimates. In this framework, the information efficiently propagates through the network to improve the overall performance [21].

In the consensus approach of the decentralized frameworks, all the agents in the network reach to a "consensus" on their estimates after collecting and processing their information locally $[15,16]$. However, this approach either requires a use of two time scales to reach to the consensus immediately or decaying learning rate for constructing the consensus among the agents in time $[16,22]$. The use of two time scales limits the performance of the network on real-time applications. On the other hand, the use of decaying learning rates hinders the ability of the system to adaptively adjust or learn in time varying environments [13].

In [14-16,23] and [24], authors present diffusion based approaches for distributed estimation, where the network is able to respond to the fast-streaming data in an online manner by using a single time scale. In the diffusion based strategies, agents process their observations locally and disclose the corresponding estimates to the neighboring nodes and improve their performance through combining the received estimates. In [13], authors prove that the diffusion based approaches outperform single time scale consensus strategies regarding the global MSE performance. However, neither of these methods consider the network topology or information disclosure procedures to obtain a globally optimal solution. On the other hand, in $[10,12]$, diffusion incremental solutions are shown to reach to the optimal estimate by defining a certain path through the network, which is not practical against the fast streaming data or the dynamic configurations.

In [25], authors presented a novel approach to obtain the teamoptimal distributed estimation of a static underlying parameter by exploiting the network structure, and the optimal information disclosure and combination without any incremental path requirements. However, in most of the real-life applications, the underlying parameter is subject to a change, i.e. it evolves in time [26]. Although there exists different studies on the distributed estimation of a dynamic parameter, these algorithms again do not consider the correlation of the disclosed information between the agents in the network due to the dynamic evolution of the underlying parameter [3,26-28]. Hence, these algorithms cannot achieve the team-optimal estimation and the problem requires a different approach than the solutions available in the literature.

To this end, we work on the team-optimal estimation of dynamic parameters over distributed networks. We first use the framework of Sayin et al. [29] to establish the model and the problem. Then, we introduce the efficient and optimal distributed learning (EODL) algorithm for the online estimation of dynamic parameters and prove that it is only applicable over certain network topologies. We also show the superior performance of the proposed method compared to the state-of-the-art methods through numerical examples.

\subsection{Main contributions}

We list our main contributions as follows:

- We show that the team optimal estimation is possible over any arbitrary network if the agents disclose the time and node stamped versions of their observations even under dynamic environments.

- We prove that the team-optimality is possible only over certain network topologies, e.g., tree networks, if the agents only disclose their local estimates.

- We introduce an algorithm for estimating a dynamic parameter that achieves the team-optimal error lower bound over these certain networks.

- We derive an efficient information sharing and combination scheme to reduce the communication load over such networks.

- We provide numerical examples to illustrate the convergence and the steady-state performance improvements achieved by our algorithm with respect to the state-of-the-art methods.

We organize the paper as follows. In Section 2, we present the team framework for the dynamic parameter estimation and show that the optimal estimate can be constructed through diffusion of the time stamped information. Then, in Section 3, we prove that the team-optimal estimation through disclosure of local estimates can be achieved only under certain network topologies. Later in Section 4, we provide an iterative algorithm to construct the optimal combination weights and the estimate over such networks. We demonstrate the performance of the proposed algorithm through series of simulations in Section 5 and conclude the paper with final remarks in Section 6.

\section{Team framework for distributed estimation}

In this paper, all random variables are represented as uppercase calligraphic letters, i.e. $\mathcal{X}$, and all the realizations of these variables are presented as their lowercase characters, i.e. $x$. All the vectors are column vectors and denoted by boldface lowercase letters.

We consider a distributed network with $m$ agents equipped with processing and communication capabilities. We form the network as an undirected graph, where vertices and edges represent the agents and the communication links respectively, as shown in Fig. 1. For each agent $i$, we denote the set of agents, whose information is available to the agent $i$ after transmission over $k$ communication links (after $k$-hops) as $\mathbf{N}_{i}^{(k)}$. We define $\mathbf{N}_{i}^{(k)}$ as

$\mathbf{N}_{i}^{(k)}=\left\{j_{1^{(k)}}, \cdots, j_{\pi_{i}^{(k)}}\right\}$

where $\pi_{i}^{(k)}=\left|\mathbf{N}_{i}^{(k)}\right|$ is the cardinality of the set $\mathbf{N}_{i}^{(k)}$. We assume that $\mathbf{N}_{i}^{(0)}=\{i\}$ and $\mathbf{N}_{i}^{(k)}=\emptyset$ for $k<0$. In Fig. 1, we demonstrate the first neighborhood of the agent $i$, where $\mathbf{N}_{i}=\left\{j_{1}, j_{2}, j_{3}\right\}$ and $\pi_{i}=3$. 


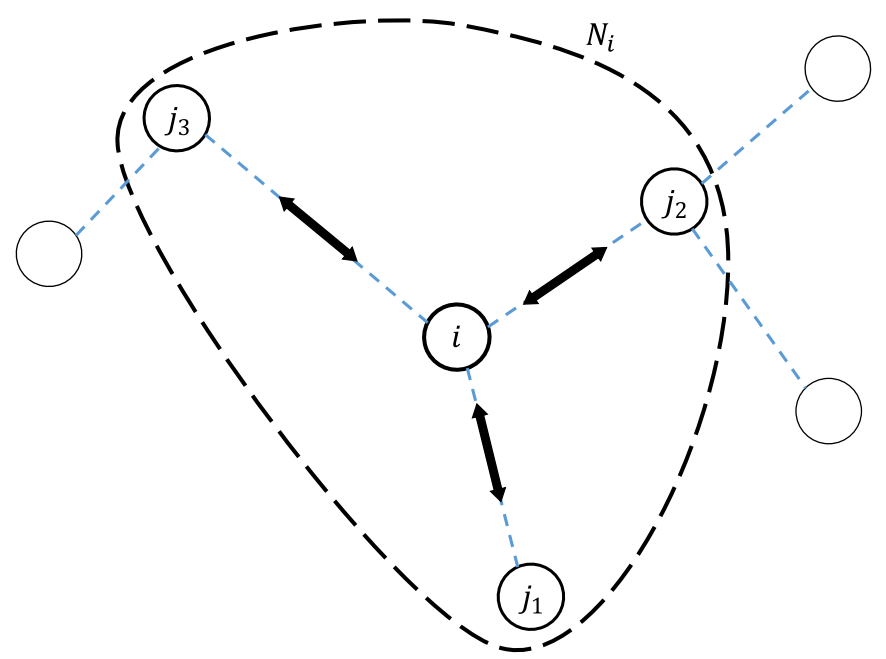

Fig. 1. First order neighborhood of the agent $i$ over a distributed network. The agent $i$ only exchanges information with the nodes in this neighborhood.

We drop the superscript on the first order neighborhood for notational simplicity.

We choose the random walk for the modeling of the underlying dynamic state since the random walk model is extensively used to model the behavior of highly complex structures from biological systems to social networks $[3,26,27]$. Hence, the underlying state $x_{t} \in \mathbb{R}$ evolves according to

$x_{t+1}=\gamma x_{t}+w_{t}$,

where $\gamma \in \mathbb{R}$ is the expected rate of change. The term $w_{t} \in \mathbb{R}$ is the state noise and it is an i.i.d. Gaussian random process $\left\{\mathcal{W}_{t}\right\}$ with variance $\sigma_{w}^{2}$. The initial state is sampled from a Gaussian random variable such that $\mathcal{X}_{0} \sim N\left(0, \sigma_{0}^{2}\right)$.

Each agent in the network observes a noisy version of the same underlying dynamic state as

$y_{i, t}=x_{t}+n_{i, t}$

for $i=1, \cdots, m$ and $n_{i, t} \in \mathbb{R}$ is a white Gaussian process $\left\{\mathcal{N}_{i, t}\right\}$ with variance $\sigma_{n_{i}}^{2}$. We assume that the observation noise is spatially independent and the variance of the noise signals are known to each agent (if they are not available, then they can be estimated from the observations [30]). Correspondingly, $y_{i, t}$ becomes a realization of a random process $\left\{\mathcal{Y}_{i, t}\right\}$, where $\mathcal{Y}_{i, t}=\mathcal{X}_{t}+\mathcal{N}_{i, t}$. At each instant, an agent receives a local observation and diffused information from the neighboring agents, while it also diffuses information to its neighboring agents.

Obviously, each agent can alone track the underlying state in the MMSE sense under certain regulatory conditions [31]. However, the use of distributed cooperation can greatly enhance the learning rate and the robustness of the system [30]. To this end, we aim to find an optimal estimation strategy regarding the MSE performance for a team of distributed agents. To provide a lower bound on the performance of the team, we first consider a case where the agents in the network disclose the stamped versions, with time and the agent ID, of their observations and the received information. Thus, each agent has access to the observations of all the other agents in the network. However, we note that only the observations from the neighboring agents can be directly received. The observations from the non-neighboring agents can only be accessed after going over certain number of communication links, i.e. the information of the agent $j \in \mathbf{N}_{i}^{(2)}$ can be accessed by the agent $i$ after being transmitted over 2 communications links. We illustrate this behavior of the distributed networks in Fig. 2, where we show $\mathbf{N}_{i}, \mathbf{N}_{i}^{(2)}$ and $\mathbf{N}_{i}^{(3)}$ for the node $i$. Only the information from $\mathbf{N}_{i}$ can be directly received by node $i$, otherwise the information have to follow the described neighborhood path to reach the node $i$.

We define the team cost of the network for a time horizon $T$, when each agent $i$ makes the estimate $\hat{x}_{i, t}$ as

$\sum_{t=1}^{T} \sum_{i=1}^{m} \mathbb{E}\left\|\mathcal{X}_{t}-\hat{x}_{i, t}\right\|^{2}$.

We also emphasize that due to the connected structure of the network, each agent will have access to all the observations in the network, although with certain delay. Therefore, we denote the information aggregated at the agent $i$ at time $t$ as

$D_{i, t}=\left\{\left\{y_{i, \tau}\right\}^{\tau \leq t},\left\{y_{j, \tau}\right\}_{j \in \mathbf{N}_{i}}^{\tau \leq t-1},\left\{y_{j, \tau}\right\}_{j \in \mathbf{N}_{i}^{(2)}}^{\tau \leq t-2}, \cdots,\left\{y_{j, \tau}\right\}_{j \in \mathbf{N}_{i}^{\left(\kappa_{i}\right)}}\right\}$,

where $\kappa_{i}$ denotes the communication link delay for the furthest node from the $i$ th node. Note that $\left\{y_{j, \tau}\right\}_{j \in \mathbf{N}^{\left(t_{i}\right)}}^{\tau \leq t-t_{i}}$ is the set of observations received from $t_{i}$ hop away neighborhood of the agent $i$, which is explicitly defined as

$$
\left\{y_{j, \tau}\right\}_{j \in \mathbf{N}^{\left(t_{i}\right)}}^{\tau \leq t t_{i}} \triangleq\left\{y_{j_{1}\left(t_{i}\right), t-t_{i}}, \ldots, y_{j_{1}\left(t_{i}\right)}, 0, \cdots, y_{\pi_{i}^{\left(t_{i}\right)}, t-t_{i}}, \ldots, y_{\pi_{i}^{\left(t_{i}\right)}, 0}\right\} .
$$

With this aggregated information, we construct the team optimization problem as

$$
\begin{aligned}
& \min _{x} \sum_{t=1}^{T} \sum_{i=1}^{m} \mathbb{E}\left[\left\|\mathcal{X}_{t}-x\right\|^{2} \mid\left\{\mathcal{Y}_{i, \tau}=y_{i, \tau}\right\}^{\tau \leq t},\right. \\
& \left.\left\{\mathcal{Y}_{j, \tau}=y_{j, \tau}\right\}_{j \leq t-1}^{\tau \leq \mathbf{N}_{i}}, \cdots,\left\{\mathcal{Y}_{j, \tau}=y_{j, \tau}\right\}_{\substack{\tau \leq t-\kappa_{i} \\
j \in \mathbf{N}_{i}^{\left(\kappa_{i}\right)}}}\right],
\end{aligned}
$$

which corresponds, for each agent, to solving

$$
\begin{aligned}
& \min _{x} \sum_{t=1}^{T} \mathbb{E}\left[\left\|\mathcal{X}_{t}-x\right\|^{2} \mid\left\{\mathcal{Y}_{i, \tau}=y_{i, \tau}\right\}^{\tau \leq t},\right. \\
& \left.\left\{\mathcal{Y}_{j, \tau}=y_{j, \tau}\right\}_{j \in \mathbf{N}_{i}}^{\tau \leq t}, \cdots,\left\{\mathcal{Y}_{j, \tau}=y_{j, \tau}\right\}_{j \in \mathbf{N}_{i}^{\left(\kappa_{i}\right)}}^{\tau \leq t-\kappa_{i}}\right] .
\end{aligned}
$$

The solution to the optimization problem in (7) at each time step $t$ gives the MMSE estimate for the agent $i$ such that

$$
\begin{aligned}
& \hat{x}_{i, t}=\mathbb{E}\left[\mathcal{X}_{t} \mid\left\{\mathcal{Y}_{i, \tau}=y_{i, \tau}\right\}^{\tau \leq t},\left\{\mathcal{Y}_{j, \tau}=y_{j, \tau}\right\}_{j \in \mathbf{N}_{i}}^{\tau \leq t-1}, \cdots,\right. \\
& \left.\left\{\mathcal{Y}_{j, \tau}=y_{j, \tau}\right\}_{j \in \mathbf{N}_{i}^{\left(\kappa_{i}\right)}}^{\tau \leq t-\kappa_{i}}\right] \text {. }
\end{aligned}
$$

Therefore, the estimate in (8) produces the team-optimal solution in an MSE sense and creates the lower bound for the teamframework.

Remark 2.1. The presented case provides a lower bound on the error performance of the team in an MSE sense through the disclosure of the time stamped observations. This scheme requires excessive amount of storage on the nodes and the communication load for the network, especially for larger networks. Note that the reduced storage and the communication load are essential for the applicability of the distributed networks to real life problems [32,33]. Therefore, we develop team-optimal estimation strategies for the distributed networks that achieves the error performance lower bound of (8), albeit the nodes only store and diffuse their current local estimates. However, in the next section, we show that such an error performance with the disclosure of local estimates can only be achieved over certain network topologies.

\section{Optimal estimation with the disclosure of local estimates}

In this section, we show that the team optimal estimation lower bound for dynamic parameters can be achieved over tree-networks 


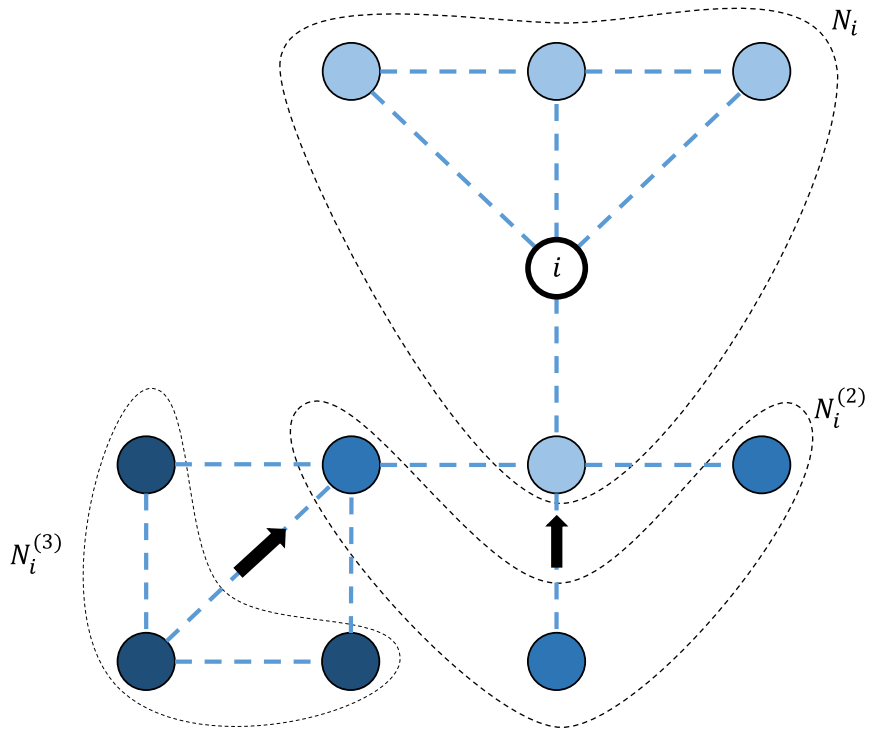

Fig. 2. Information from the agent in $\mathbf{N}_{i}$ can be directly received by the node $i$. Information coming from the agents in $\mathbf{N}_{i}^{(2)}$ and $\mathbf{N}_{i}^{(3)}$ can be accessed with a certain delay.

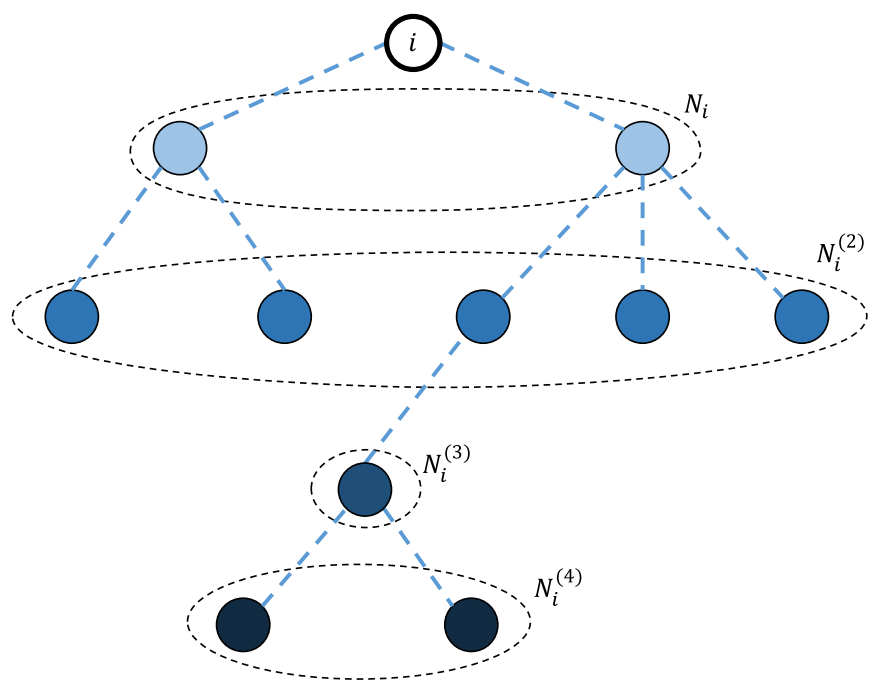

Fig. 3. Structure of a depth- 4 tree network with the corresponding neighborhoods of the agent $i$. Note that we eliminated the cyclic connections from Fig. 2 to avoid multipath information diffusion and obtain team-optimal estimation.

through disclosure of local estimates and such performance cannot be achieved over cyclic networks [29]. We define the tree-networks as graph structures, where the vertices are connected with undirected edges without any cycles as shown in Fig. 3. We also note that for any arbitrary network topology, a minimum spanning tree of the network can be constructed by eliminating the cycles [34-37].

Using the tree structure of the network, we partition the set of information coming from a particular neighborhood. For the tree networks, a neighboring set for the agent $i$ can be expressed as

$\mathbf{N}_{i}^{(k)}=\bigcup_{j \in \mathbf{N}_{i}}\left(\mathbf{N}_{i}^{(k)} \cap \mathbf{N}_{j}^{(k-1)}\right)$

and again due to the network structure, the intersecting sets are disjoint such that

$\left(\mathbf{N}_{i}^{(k)} \cap \mathbf{N}_{j_{1}}^{(k-1)}\right) \bigcap\left(\mathbf{N}_{i}^{(k)} \cap \mathbf{N}_{j_{2}}^{(k-1)}\right)=\emptyset$ for all $j_{1}, j_{2} \in \mathbf{N}_{i}$ and $j_{1} \neq j_{2}$. Therefore, we partition the information received at the agent $i$ after $k$-hops as

$\left\{y_{j, \tau}\right\}_{j \in \mathbf{N}_{i}^{(k)}}^{\tau \leq t-k}=\left\{\left\{y_{j, \tau}\right\}_{j \in \mathbf{N}_{i}^{(k)} \cap \mathbf{N}_{j_{1}}^{(k-1)}}^{\tau \leq t-k}, \cdots,\left\{y_{j, \tau}\right\}_{j \in \mathbf{N}_{i}^{(k)} \cap \mathbf{N}_{j_{\pi_{i}}}^{(k-1)}}^{\tau \leq t-k}\right\}$.

Using this partitioning method, we define the set of new measurements coming from agent $j$ to $i$ at time $t=2$ as

$z_{j \rightarrow i, 2} \triangleq\left\{\left\{y_{k, \tau}\right\}_{k \in \mathbf{N}_{i} \cap \mathbf{N}_{j}^{(0)}},\left\{y_{k, \tau}\right\}_{k \in \mathbf{N}_{i}^{(2)} \cap \mathbf{N}_{j}^{(1)}}\right\}$.

Note that the expression in (9) can also be written as

$z_{j \rightarrow i, 2}=D_{j, 2} /\left\{y_{j, 1}, y_{i, 1}\right\}$

where $y_{j, 1}=D_{j, 1}$ and $y_{i, 1}=D_{i, 1}=z_{i \rightarrow j, 1}$. Thus we can generalize the new information expression for any time $t$ as

$z_{j \rightarrow i, t}=D_{j, t} /\left\{D_{j, t-1} \cup z_{i \rightarrow j, t-1}\right\}$.

Using (11), we write all the information aggregated at the agent $i$ as

$D_{i, t}=\left\{y_{i, t}, z_{j_{1} \rightarrow i, t-1}, \cdots, z_{j_{\pi_{i}} \rightarrow i, t-1}, D_{i, t-1}\right\}$,

where $z_{j \rightarrow i, t}$ is constructible from $D_{i, \tau}$ and $D_{j, \tau}$ for $\tau \leq t$ using (10) and (11) as follows

$z_{j \rightarrow i, t}=D_{j, t} /\left\{D_{j, t-1} \cup\left\{D_{i, t-1} /\left\{D_{i, t-2} \cup z_{j \rightarrow i, t-2}\right\}\right\}\right\}$.

Therefore, using (12), we construct the optimal estimate again with an abuse of notation as

$$
\begin{aligned}
\hat{x}_{i, t} & =\mathbb{E}\left[\mathcal{X}_{t} \mid D_{i, t}\right] \\
& =\mathbb{E}\left[\mathcal{X}_{t} \mid y_{i, t}, z_{j_{1} \rightarrow i, t-1}, \cdots, z_{j_{\pi_{i}} \rightarrow i, t-1}, D_{i, t-1}\right] .
\end{aligned}
$$

Considering $z_{j \rightarrow i, t}$ is constructible from $D_{i, \tau}$ and $D_{j, \tau}$ for $\tau \leq t$ as in (13), we write the optimal estimate in (14) as

$$
\begin{aligned}
\hat{x}_{i, t} & =\mathbb{E}\left[\mathcal{X}_{t} \mid\left\{y_{i}, \tau\right\}^{\tau \leq t},\left\{D_{j, \tau}\right\}_{j \in \mathbf{N}_{i}}^{\tau \leq t-1}\right] \\
& =\mathbb{E}\left[\mathcal{X}_{t} \mid y_{i, t}, D_{i, t-1},\left\{D_{j, t-1}\right\}_{j \in \mathbf{N}_{i}}\right]
\end{aligned}
$$

and since $\hat{x}_{j, t-1}=\mathbb{E}\left[\mathcal{X}_{t-1} \mid D_{j, t-1}\right]$, we obtain

$$
\begin{aligned}
\hat{x}_{i, t} & =\mathbb{E}\left[\mathcal{X}_{t} \mid y_{i, t}, \mathbb{E}\left[\mathcal{X} \mid D_{i, t-1}\right],\left\{\mathbb{E}\left[\mathcal{X} \mid D_{j, t-1}\right]\right\}_{j \in \mathbf{N}_{i}}\right] \\
& =\mathbb{E}\left[\mathcal{X}_{t} \mid y_{i, t}, \hat{x}_{i, t-1},\left\{\hat{x}_{j, t-1}\right\}_{j \in \mathbf{N}_{i}}\right] .
\end{aligned}
$$

Hence, we conclude that we can construct the optimal estimate through disclosure of local estimates over the tree-networks.

In the following, we introduce the efficient and the optimal distributed online learning algorithm for dynamic state estimation. We propose a method that iteratively constructs the team-optimal estimate in (15) for dynamic parameters and achieves the error lower bound in (6).

\section{Efficient and optimal distributed online learning}

In Section 3, we show that over a tree network, the teamoptimal estimate can be constructed using the disclosure of local estimates as

$\hat{x}_{i, t}=\mathbb{E}\left[\mathcal{X}_{t} \mid y_{i, t}, \hat{x}_{i, t-1},\left\{\hat{x}_{j, t-1}\right\}_{j \in \mathbf{N}_{i}}\right]$.

Each local estimate $\hat{x}_{i, t}$ is linear in previous estimates $\hat{x}_{i, t-1}$ and $\left\{\hat{x}_{j, t-1}\right\}_{j \in \mathbf{N}_{i}}$. Therefore, instead of disclosing the local estimates, we constrain each agent to disclose the information that was not included in the old estimates. Then each agent extracts only the innovation terms, i.e. the new information in the disclosed data that 
the agent has not received before. Although this operation imposes more computational load on the agents, it significantly reduces the communication load on the network, which is more essential for highly-connected larger networks that require more power for the transmission of information [32].

We denote the innovation term extracted at the agent $i$ from the data disclosed by the agent $j$ at time $t$ as $z_{j \rightarrow i, t-1}$. With this definition, we define the random vector collecting the previous estimate and the aggregated information on the agent $i$ at time $t$ as

$d_{i, t}=\left[\begin{array}{llllll}\mathcal{Y}_{i, t} & \hat{\mathcal{X}}_{i, t-1} & \mathcal{Z}_{j_{1} \rightarrow i, t-1} & \cdots & \mathcal{Z}_{j_{\pi_{i}} \rightarrow i, t-1}\end{array}\right]^{T}$,

so that we find the optimal estimate of the state with realizations of the elements in $\boldsymbol{d}_{i, t}$ as

$\hat{x}_{i, t}=\mathbb{E}\left[\mathcal{X}_{t} \mid \mathcal{Y}_{i, t}=y_{i, t}, \hat{\mathcal{X}}_{i, t-1}=\hat{x}_{i, t-1},\left\{\mathcal{Z}_{j \rightarrow i, t-1}=z_{j \rightarrow i, t-1}\right\}_{j \in \mathbf{N}_{i}}\right]$.

Due to the state-space model defined in (2) and (3), all the parameters in (16) are jointly Gaussian. Hence, for the estimation of the next state at the agent $i$, we have

$\hat{x}_{i, t}=\alpha_{i, t} \hat{x}_{i, t-1}+\beta_{i, t} y_{i, t}+\sum_{j \in \mathbf{N}_{i}} c_{i, t}^{(j)} z_{j \rightarrow i, t-1} m$

where $\alpha_{i, t}, \beta_{i, t}$, and $c_{i, j}^{(j)}$ are the scalar coefficients to represent the estimation as the linear combination of the parameters in $\boldsymbol{d}_{i, t}$. Thus, they are the unknown parameters of our algorithm to be estimated and we provide the estimation procedure for them in Algorithm 1.

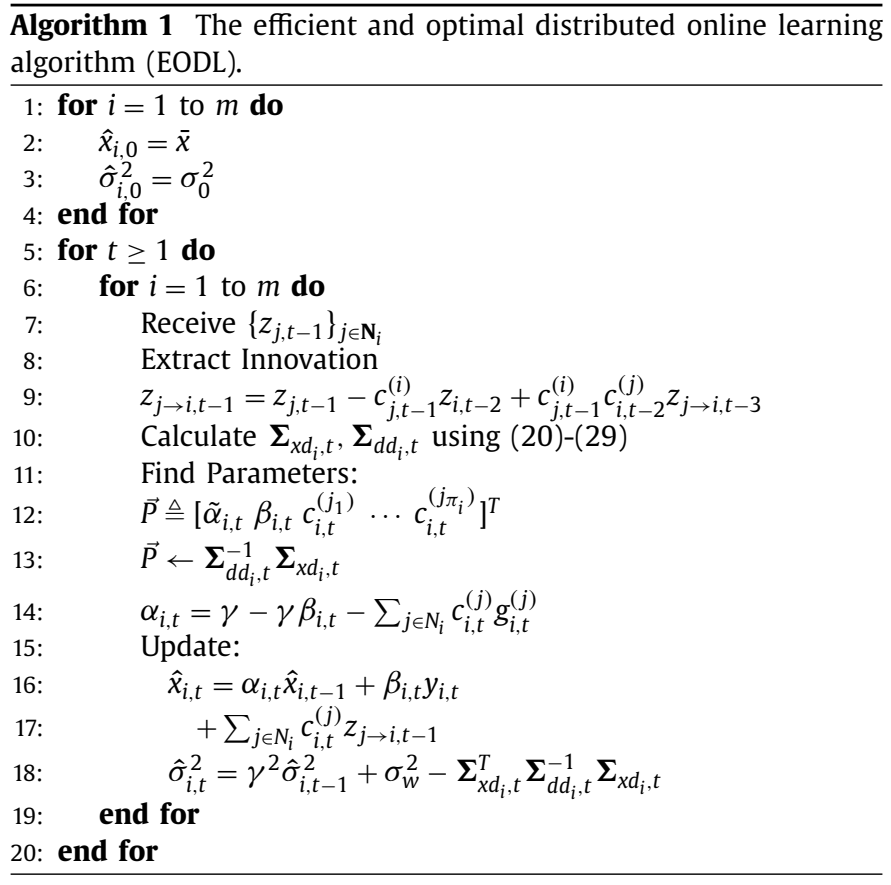

Using the estimation equation in (17), the information disclosed by the agent $j$ at time $t$ is given by

$$
\begin{aligned}
z_{j, t} & =\hat{x}_{j, t}-\alpha_{j, t} \hat{x}_{j, t-1} \\
& =\beta_{j, t} y_{j, t}+\sum_{k \in \mathbf{N}_{j}} c_{j, t}^{(k)} z_{k \rightarrow j, t-1} .
\end{aligned}
$$

Hence, we extract the innovation from the disclosed information on the agent $i$ as

$$
\begin{aligned}
z_{j \rightarrow i, t} & =z_{j, t}-c_{j, t}^{(i)} z_{i \rightarrow j, t-1} \\
& =z_{j, t}-c_{j, t}^{(i)} z_{i, t-1}+c_{j, t}^{(i)} c_{i, t-1}^{(j)} z_{j \rightarrow i, t-2} .
\end{aligned}
$$

Remark 4.1. Some of the previously diffused information are received after certain delays over the network due to multi-hops. Therefore, some of the received information will be the noisy versions of the previous instances of the underlying state. Due to the random walk model in (2), the state noise on these previous instances will become correlated with the more recent observations. Hence, this situation requires a significantly more detailed approach than the existing methods [25].

In order to calculate the parameters in the estimation recursion (17), we first need to calculate the auto-correlation matrix of $\boldsymbol{d}_{i, t}$ and the cross-correlation vector with the underlying state $\mathcal{X}_{t}$, where we define them as $\boldsymbol{\Sigma}_{d d_{i}, t}$ and $\boldsymbol{\Sigma}_{x d_{i}, t}$ respectively. We first calculate the terms of $\boldsymbol{\Sigma}_{x d_{i}, t}$ starting with

$$
\begin{aligned}
\mathbb{E}\left[\mathcal{X}_{t} \mathcal{Y}_{i, t}\right] & =\mathbb{E}\left[\mathcal{X}_{t}\left(\mathcal{X}_{t}+\mathcal{N}_{i, t}\right)\right]=\mathbb{E}\left[\mathcal{X}_{t}^{2}\right] \\
& =\gamma^{2} \mathbb{E}\left[\mathcal{X}_{t-1}^{2}\right]+\sigma_{w}^{2} .
\end{aligned}
$$

Then, we calculate

$$
\begin{aligned}
\mathbb{E}\left[\mathcal{X}_{t} \hat{\mathcal{X}}_{i, t-1}\right]= & \mathbb{E}\left[\mathcal { X } _ { t } \left(\alpha_{i, t-1} \hat{\mathcal{X}}_{i, t-2}\right.\right. \\
& \left.\left.+\beta_{i, t-1} \mathcal{Y}_{i, t-1}+\sum_{j \in \mathbf{N}_{i}} c_{i, t-1}^{(j)} \mathcal{Z}_{j \rightarrow i, t-2}\right)\right] \\
= & \alpha_{i, t-1} \mathbb{E}\left[\mathcal{X}_{t} \hat{\mathcal{X}}_{i, t-2}+\beta_{i, t-1} \mathbb{E}\left[\mathcal{X}_{t} \mathcal{Y}_{i, t-1}\right]\right] \\
& +\sum_{j \in \mathbf{N}_{i}} c_{i, t-1}^{(j)} \mathbb{E}\left[\mathcal{X}_{t} \mathcal{Z}_{j \rightarrow i, t-2}\right] \\
= & \gamma^{2} \alpha_{i, t-1} \mathbb{E}\left[\mathcal{X}_{t-2} \hat{\mathcal{X}}_{i, t-2}\right]+\gamma \beta_{i, t-1} \mathbb{E}\left[\mathcal{X}_{t-1}^{2}\right] \\
& +\sum_{j \in \mathbf{N}_{i}} c_{i, t-1}^{(j)} \mathbb{E}\left[\mathcal{X}_{t} \mathcal{Z}_{j \rightarrow i, t-2}\right] .
\end{aligned}
$$

In order to calculate (21), we also need to calculate $\mathbb{E}\left[\mathcal{X}_{t} \mathcal{Z}_{j \rightarrow i, t-2}\right]$. For that, we first introduce

$h_{i, 0}=\gamma\left[\begin{array}{c}\beta_{j_{1}, 0} \\ \vdots \\ \beta_{j_{\pi_{i}}, 0}\end{array}\right] \mathbb{E}\left[\mathcal{X}_{0}^{2}\right]$.

Then, with this initialization, for any time $t$, we find

$$
\begin{aligned}
h_{i, t}= & \gamma\left[\begin{array}{c}
\beta_{j_{1}, t} \mathbb{E}\left[\mathcal{X}_{t}^{2}\right]+c_{j_{1}, t}^{T} h_{j_{1}, t-1} \\
\vdots \\
\beta_{j_{\pi_{i}}, t} \mathbb{E}\left[\mathcal{X}_{t}^{2}\right]+c_{j_{\pi_{i}}, t}^{T} h_{j_{\pi_{i}}, t-1}
\end{array}\right]-\gamma\left[\begin{array}{c}
c_{j_{1}, t}^{(i)} \\
\vdots \\
c_{j_{\pi_{i}}, t}^{(i)}
\end{array}\right] \\
\odot & {\left[\begin{array}{c}
h_{j_{1}, t-1}^{(i)} \\
\vdots \\
h_{j_{\pi_{i}, t-1}^{(i)}}
\end{array}\right], }
\end{aligned}
$$

where

$c_{j_{1}, t}=\left[c_{j_{1}, 1}^{\left(k_{1}\right)} \ldots c_{j_{1}, 1}^{\left(k_{\pi_{1}}\right)}\right]^{T}, \quad k \in \mathbf{N}_{j_{1}}$.

Note that $\boldsymbol{h}_{i, t}$ can also be expressed as

$h_{i, t-1}=\left[\begin{array}{c}E\left[\mathcal{Z}_{j_{1} \rightarrow i, t-1} \mathcal{X}_{t}\right] \\ \vdots \\ E\left[\mathcal{Z}_{j_{\pi_{i}} \rightarrow i, t-1} \mathcal{X}_{t}\right]\end{array}\right]$.

Using this notation, we obtain

$$
\begin{aligned}
\mathbb{E}\left[\mathcal{X}_{t} \mathcal{Z}_{j \rightarrow i, t-2}\right] & =\gamma \mathbb{E}\left[\mathcal{X}_{t-1} \mathcal{Z}_{j \rightarrow i, t-2}\right] \\
& =\gamma h_{i, t-1}^{(j)} .
\end{aligned}
$$

Therefore, we can finalize the calculation of $\mathbb{E}\left[\mathcal{X}_{t} \hat{\mathcal{X}}_{t-1}\right]$ as $\mathbb{E}\left[\mathcal{X}_{t} \hat{\mathcal{X}}_{t-1}\right]=\gamma^{2} \alpha_{i, t-1} \mathbb{E}\left[\mathcal{X}_{t-2} \hat{\mathcal{X}}_{t-2}\right]+\gamma \beta_{i, t-1} \mathbb{E}\left[\mathcal{X}_{t-1}^{2}\right]+\gamma c_{i, t-1}^{T} h_{i, t-1}$. 
Additionally, we define the cross correlation term between the state and the estimate as

$\tilde{\sigma}_{i, t}^{2} \triangleq \mathbb{E}\left[\mathcal{X}_{t} \hat{\mathcal{X}}_{i, t}\right]$

$$
=\gamma \alpha_{i, t} \tilde{\sigma}_{i, t-1}^{2}+\beta_{i, t} \mathbb{E}\left[\mathcal{X}_{t}^{2}\right]+c_{i, t}^{T} h_{i, t}
$$

and the variance for the underlying state as

$\sigma_{t}^{2} \triangleq \mathbb{E}\left[\mathcal{X}_{t}^{2}\right]$

$$
=\gamma^{2} \sigma_{t-1}^{2}+\sigma_{w}^{2}
$$

which concludes our calculation for the terms in $\boldsymbol{\Sigma}_{x d_{i}, t}$ such that

$$
\begin{aligned}
\boldsymbol{\Sigma}_{x d_{i}, t} & =\left[\mathbb{E}\left[\mathcal{X}_{t} \mathcal{Y}_{i, t}\right] \mathbb{E}\left[\mathcal{X}_{t} \hat{\mathcal{X}}_{i, t-1}\right] \mathbb{E}\left[\mathcal{X}_{t} \mathcal{Z}_{j_{1} \rightarrow i, t-1}\right] \cdots \mathbb{E}\left[\mathcal{X}_{t} \mathcal{Z}_{j_{\pi_{i}} \rightarrow i, t-1}\right]\right]^{T} \\
& =\left[\begin{array}{lll}
\gamma^{2} \sigma_{t-1}^{2}+\sigma_{w}^{2} & \gamma \tilde{\sigma}_{i, t-1}^{2} & h_{i, t-1}^{T}
\end{array}\right]^{T} .
\end{aligned}
$$

Next, we calculate the terms of $\boldsymbol{\Sigma}_{d d_{i}, t}$. First, we have

$$
\begin{aligned}
\mathbb{E}\left[\mathcal{Y}_{i, t}^{2}\right] & =\mathbb{E}\left[\left(\mathcal{X}_{t}+\mathcal{N}_{i, t}\right)^{2}\right] \\
& =\sigma_{t}^{2}+\sigma_{n_{i}}^{2} .
\end{aligned}
$$

Then, for the term $\mathbb{E}\left[\hat{\mathcal{X}}_{i, t-1} \mathcal{Y}_{i, t}\right]$ we get

$$
\begin{aligned}
\mathbb{E}\left[\hat{\mathcal{X}}_{i, t-1} \mathcal{Y}_{i, t}\right] & =\mathbb{E}\left[\hat{\mathcal{X}}_{i, t-1} \mathcal{X}_{t}\right] \\
& =\gamma \tilde{\sigma}_{i, t-1}^{2}
\end{aligned}
$$

and note that we already found that $\mathbb{E}\left[\mathcal{Y}_{i, t} \mathcal{Z}_{j \rightarrow i, t-1}\right]=h_{i, t-1}^{(j)}$. We then calculate the terms that include the random variable corresponding to the estimate of the previous state. We begin with defining

$$
\begin{aligned}
\hat{\sigma}_{i, t-1}^{2} \triangleq & \mathbb{E}\left[\hat{\mathcal{X}}_{i, t-1}^{2}\right] \\
= & \mathbb{E}\left[\left(\alpha_{i, t-} \hat{\mathcal{X}}_{i, t-2}+\beta_{i, t-2} \mathcal{Y}_{i, t-1}+\sum_{j \in \mathbf{N}_{i}} c_{i, t-1}^{(j)} \mathcal{Z}_{j \rightarrow i, t-2}\right)^{2}\right] \\
= & \alpha_{i, t-1}^{2} \underbrace{\mathbb{E}\left[\hat{\mathcal{X}}_{i, t-2}^{2}\right]}_{\hat{\sigma}_{i, t-2}^{2}}+2 \alpha_{i, t-1} \beta_{i, t-2} \underbrace{\mathbb{E}\left[\hat{\mathcal{X}}_{i, t-2} \mathcal{Y}_{i, t-1}\right]}_{\gamma \tilde{\sigma}_{i, t-2}^{2}} \\
& +2 \alpha_{i, t-1} \sum_{j \in N_{i}} c_{i, t-1}^{(j)} E\left[\hat{\mathcal{X}}_{i, t-2} \mathcal{Z}_{j \rightarrow i, t-2}\right]+\beta_{i, t-2}^{2} \underbrace{\mathbb{E}\left[\mathcal{Y}_{i, t-1}^{2}\right]}_{\sigma_{t-1}^{2}+\sigma_{n_{i}}^{2}} \\
& +2 \beta_{i, t-2} \sum_{j \in \mathbf{N}_{i}} c_{i, t-1}^{(j)} \mathbb{E}\left[\mathcal{Y}_{i, t-1} \mathcal{Z}_{j \rightarrow i, t-2}\right] \\
& +\mathbb{E}\left[\left(\sum_{j \in \mathbf{N}_{i}} c_{i, t-1}^{(j)} \mathcal{Z}_{j \rightarrow i, t-2} h_{i, t-2}\right.\right.
\end{aligned}
$$

and $\hat{\sigma}_{i, 0}^{2}=\beta_{i, 0}^{2}\left(\sigma_{0}^{2}+\sigma_{n_{i}}^{2}\right)$. We need to calculate $\mathbb{E}\left[\hat{\mathcal{K}}_{i, t-2} \mathcal{Z}_{j \rightarrow i, t-2}\right]$ in order to complete the calculation of (24). For that, we introduce a more compact form of the term $\mathcal{Z}_{j \rightarrow i, t}$ in (25), where in the following, $\kappa_{i}$ is the number of hops from the furthest agent and (i.n.t.) is the abbreviation of independent noise terms. We point out that the term $g_{i, t}^{(j)}$ can be calculated in a recursive form as

$$
\begin{aligned}
\mathcal{Z}_{j \rightarrow i, t}= & \beta_{j, t} \mathcal{Y}_{j, t}+\sum_{k \in \mathbf{N}_{j}, k \neq i} c_{j, t}^{(k)}\left(\beta_{k, t-1} \mathcal{Y}_{k, t-1}+\sum_{l \in \mathbf{N}_{k}, l \neq j} c_{k, t-1}^{(l)}\left(\beta_{l, t-2} \mathcal{Y}_{l, t-2}+\sum \cdots\right)\right) \\
= & {[\overbrace{\beta_{j, t}+\frac{1}{\gamma} \sum_{k \in \mathbf{N}_{j}, k \neq i} c_{j, t}^{(k)}\left(\beta_{k, t-1}+\frac{1}{\gamma} \sum_{l \in \mathbf{N}_{k}, l \neq j} c_{k, t-1}^{(l)}\left(\beta_{l, t-2}+\frac{1}{\gamma} \sum \cdots\right)\right)}^{g_{i, t}^{(j)}}] \mathcal{X}_{t} } \\
& -\left[\frac{1}{\gamma} \sum_{k \in \mathbf{N}_{j}, k \neq i} c_{j, t}^{(k)}\left(\beta_{k, t-1}+\frac{1}{\gamma} \sum_{l \in \mathbf{N}_{k}, l \neq j} c_{k, t-1}^{(l)}\left(\beta_{l, t-2}+\frac{1}{\gamma} \sum \cdots\right)\right)\right] \mathcal{W}_{t-1}
\end{aligned}
$$

$$
\begin{aligned}
& -\left[\frac{1}{\gamma} \sum_{k \in N_{j}, k \neq i l \in N_{k}, l \neq j} c_{j, t}^{(k)} c_{k, t-1}^{(l)}\left(\beta_{l, t-2}+\frac{1}{\gamma} \sum \cdots\right)\right] \\
& \left.\mathcal{W}_{t-2}-\cdots-[\cdots] \mathcal{W}_{t-\kappa_{i}+1}+\text { (i.n.t. }\right) .
\end{aligned}
$$

Hence, using (25), we write the term $\mathcal{Z}_{j \rightarrow i, t}$ as

$$
\begin{aligned}
\mathcal{Z}_{j \rightarrow i, t}= & g_{i, t}^{(j)} \mathcal{X}_{t}-\left(g_{i, t}^{(j)}-\beta_{j, t}\right) \mathcal{W}_{t-1} \\
& -\gamma\left(g_{i, t}^{(j)}-\beta_{j, t}-\frac{1}{\gamma} \sum_{k \in N_{j}, k \neq i} c_{j, t}^{(k)} \beta_{k, t-1}\right) \mathcal{W}_{t-2} \\
& -\cdots-(\cdots) \mathcal{W}_{t-\kappa_{i}+1}+\text { (i.n.t.) }
\end{aligned}
$$

and we obtain

$\mathbb{E}\left[\hat{\mathcal{X}}_{i, t} \mathcal{Z}_{j \rightarrow i, t}\right]=g_{i, t}^{(j)} \mathbb{E}\left[\hat{\mathcal{X}}_{i, t} \mathcal{X}_{t}\right]-\left(g_{i, t}^{(j)}-\beta_{j, t}\right) \mathbb{E}\left[\hat{\mathcal{X}}_{i, t} \mathcal{W}_{t-1}\right]$

$$
-\cdots-(\cdots) \mathbb{E}\left[\hat{\mathcal{X}}_{i, t} \mathcal{W}_{t-\kappa_{i}+1}\right],
$$

where the coefficients are calculated recursively.

The state noise $\mathcal{W}_{t}$ is independent from previous states and we express the term $\hat{\mathcal{X}}_{i, t}$ as

$$
\begin{aligned}
\hat{\mathcal{X}}_{i, t}= & \beta_{i, t} \mathcal{W}_{t-1}+\left(\alpha_{i, t} \beta_{i, t-1}+\sum_{j \in N_{i}} c_{i, t}^{(j)} \beta_{j, t-1}\right) \mathcal{W}_{t-2} \\
& +\left(\alpha_{i, t} \alpha_{i, t-1} \beta_{i, t-2}+\alpha_{i, t} \sum_{j \in N_{i}} c_{i, t}^{(j)} \beta_{j, t-2}\right. \\
& \left.+\sum_{j \in N_{i}} \sum_{k \in N_{j}, k \neq i} c_{i, t}^{(j)} c_{j, t-1}^{(k)} \beta_{k, t-2}\right) \mathcal{W}_{t-3} \\
& +\cdots+(\cdots) \mathcal{W}_{t-\kappa_{i}+1}+\text { i.n.t. }
\end{aligned}
$$

Therefore, we conclude that

$\mathbb{E}\left[\hat{\mathcal{X}}_{i, t} \mathcal{Z}_{j \rightarrow i, t}\right]=g_{i, t}^{(j)} \mathbb{E}\left[\hat{\mathcal{X}}_{i, t} \mathcal{X}_{t}\right]+A \sigma_{w}^{2}$,

where $A$ is calculated according to the recursions in (26) and (27).

Finally, we calculate the remaining terms of $\boldsymbol{\Sigma}_{d d_{i}, t}$ as

$$
\begin{aligned}
\mathbb{E} & {\left[\left(\mathcal{Z}_{j \rightarrow i, t-1}\right)^{2}\right]=\left(g_{i, t-1}^{(j)^{2}}\right) \sigma_{t-1}^{2}+\left[\left(\left(g_{i, t-1}^{(j)}-\beta_{j, t-1}\right)^{2}\right.\right.} \\
& \left.-\left(g_{i, t-1}^{(j)}-\beta_{j, t-1}-\frac{1}{\gamma} \sum_{k \in N_{j}, k \neq i} c_{j, t-1}^{(k)} \beta_{k, t-2}\right)\right)^{2} \\
& \left.-B_{j, 4}^{2}-B_{j, 5}^{2} \cdots-B_{j, \kappa_{i}}^{2}\right] \sigma_{w}^{2} \\
& +\left(\beta_{j, t-1}\right)^{2} \sigma_{n_{j}}+\sum_{k \in N_{j}, k \neq i}\left(c_{j, t-1}^{(k)} \beta_{k, t-2}\right) \sigma_{n_{k}}^{2} \\
& +\sum_{k \in N_{j}, k \neq i} \sum_{\left(c_{j \in N_{k}, l \neq j}^{(k)} c_{j, t}^{(l)} c_{k, t-2} \beta_{k, t-2} \beta_{l, t-3}\right)^{2} \sigma_{n_{l}}^{2}+\cdots}+\sum_{k \in N_{j}, k \neq i} \sum_{\cdots \in N_{k}, l \neq j} \sum_{r \in N_{i}^{\left(k_{i-3}\right)}} \sum_{s \in N_{i}^{\left(k_{i-2}\right)}} \sum_{m \in N_{i}^{\left(k_{i-1}\right)}, m \neq r} \\
& \left(c_{j, t-1}^{(k)} c_{k, t-2}^{(l)} \cdots c_{s, t-\kappa_{i}}^{(m)} \beta_{k, t-2} \beta_{l, t-3} \cdots \beta_{m, t-\kappa_{i}}\right)^{2} \sigma_{m}^{2}
\end{aligned}
$$

and

$$
\begin{aligned}
& \mathbb{E}\left[\mathcal{Z}_{j_{o} \rightarrow i, t-1} \mathcal{Z}_{j_{p} \rightarrow i, t-1}\right]=g_{i, t-1}^{j_{o}} g_{i, t-1}^{j_{p}} \sigma_{t-1}^{2} \\
& +\left[\left(g_{i, t-1}^{\left(j_{o}\right)}-\beta_{j_{o}, t-1}\right)\left(g_{i, t-1}^{\left(j_{p}\right)}-\beta_{j_{p}, t-1}\right)\right. \\
& -\left(g_{i, t-1}^{\left(j_{o}\right)}-\beta_{j_{o}, t-1}-\frac{1}{\gamma} \sum_{k \in N_{j_{o}}, k \neq i} c_{j_{o}, t-1}^{(k)} \beta_{k, t-2}\right) \\
& \left(g_{i, t-1}^{\left(j_{p}\right)}-\beta_{j_{p}, t-1}-\frac{1}{\gamma} \sum_{k \in N_{j_{p}}, k \neq i} c_{j_{p}, t-1}^{(k)} \beta_{k, t-2}\right) \\
& \left.-B_{j_{o}, 4} B_{j_{p}, 4}-\cdots-B_{j_{o}, K_{i}} B_{j_{p}, \kappa_{i}}\right] \sigma_{w}^{2},
\end{aligned}
$$


Table 1

Computational complexities of the algorithms. Here, $d$ represents the dimensionality of the state for vector dynamic models.

\begin{tabular}{lll}
\hline Algorithm & Scalar case complexity & Vector case complexity \\
\hline EODL & $O\left(m \pi^{3}\right)$ & $O\left(m \pi^{3} d\right)$ \\
D-LMS & $O(m \pi)$ & $O(m \pi d)$ \\
D-RLS & $O(m \pi)$ & $O\left(m \pi d^{2}\right)$ \\
D-Kalman & $O(m \pi)$ & $O\left(m \pi d^{3}\right)$ \\
\hline
\end{tabular}

where $o, p \in\left\{1, \ldots, \pi_{i}\right\}, o \neq p$ and $B_{j, t}$ for $t=4, \ldots, \kappa-i$ represents the remaining recursive terms derived in (26).

Next, we recursively calculate the parameters in (17). We define the vector containing the parameters as

$P \triangleq\left[\begin{array}{lllll}\tilde{\alpha}_{i, t} & \beta_{i, t} & c_{i, t}^{\left(j_{1}\right)} & \cdots & c_{i, t}^{\left(j_{\pi_{i}}\right)}\end{array}\right]^{T}$.

In (14), all the conditioned parameters are jointly Gaussian with the state. Therefore, we calculate the parameter vector as $P=$ $\boldsymbol{\Sigma}_{d d_{i}, t}^{-1} \boldsymbol{\Sigma}_{x d_{i}, t}$. The estimation and the variance recursions are given by

$\hat{x}_{i, t}=\gamma \hat{x}_{i, t-1}+\beta_{i, t}\left(y_{i, t}-\gamma \hat{x}_{i, t-1}\right)+\sum_{j \in N_{i}} c_{i, t}^{(j)}\left(z_{j \rightarrow i, t-1}-g_{i, t}^{(j)} \hat{x}_{i, t-1}\right)$,

$\hat{\sigma}_{i, t}^{2}=\gamma^{2} \hat{\sigma}_{i, t-1}^{2}+\sigma_{w}^{2}-\boldsymbol{\Sigma}_{x d_{i}, t}^{T} \boldsymbol{\Sigma}_{d d_{i}, t}^{-1} \boldsymbol{\Sigma}_{x d_{i}, t}$.

Hence, for the parameter $\alpha_{i, t}$ in (17), we have

$\alpha_{i, t}=\gamma-\gamma \beta_{i, t}-\sum_{j \in N_{i}} c_{i, t}^{(j)} g_{i, t}^{(j)}$,

which finalizes the efficient and optimal distributed online learning algorithm. We give the detailed pseudo-code of the overall algorithm in Algorithm 1. Here, the computational complexity of our algorithm is dominated by the matrix inversion on line 13 and 18. Thus, we achieve $O\left(\pi_{i}^{3}\right)$ complexity for each agent $i$, where $\pi_{i}$ determines the order of the size of $\boldsymbol{\Sigma}_{d d_{i}, t}$. If we assume that each agent $i$ has $\pi=\frac{1}{m} \sum_{j=1}^{m} \pi_{j}$ neighbors on the average, then our algorithm has $O\left(m \pi^{3}\right)$ complexity in total. Since we deal with a scalar parameter case, i.e., $x_{t} \in \mathbb{R}$, D-LMS, D-RLS and D-Kalman algorithms need only $O(m \pi)$ complexity as illustrated in Table 1 $[12,14,24]$. However, as the data size increases, e.g., assume that $x_{t} \in \mathbb{R}^{d}$, the complexities change as shown in Table 1 . In Table 1 , we observe only $O(d)$ factors for the LMS and EODL algorithms. However, the RLS algorithm causes a $O\left(d^{2}\right)$ factor due to the update of its correlation matrix [12] and the Kalman filtering algorithm causes a $O\left(d^{3}\right)$ factor due to the matrix inversion in its implementation [14]. Thus, our algorithm (EODL) achieves significant complexity reductions especially for sparsely connected networks, i.e., $\pi$ is small, and high dimensional applications, i.e., the data size $d$ is high, compared to the conventional algorithms.

In the following, we provide numerical examples to evaluate the performance of our algorithm against several other distributed estimation algorithms under different scenarios.

Remark 4.2. Scalar dynamic parameter models are extensively studied since they model a wide range of real life applications $[3,26,27]$. Thus, we work on a scalar dynamic model in this paper. However, our approach can be straightforwardly extended to a vector dynamic model case as in the following.

We define our vector dynamic model as follows

$x_{t+1}=\gamma x_{t}+w_{t}$,

where $\gamma \in \mathbb{R}$ is the expected rate of change. The term $w_{t} \in \mathbb{R}^{p}$ is the state noise and it is an i.i.d. Gaussian random process $\left\{\mathcal{W}_{t}\right\}$ with covariance $\boldsymbol{\Sigma}_{w}$. The initial state is sampled from a Gaussian random variable such that $\mathcal{X}_{0} \sim N\left(0, \Sigma_{0}\right)$. Similarly, the observation model in (3) and its definitions change according to (30). Moreover, (16) becomes an information matrix due to (30). After this change, we follow the same procedure in (20)-(29) based on the new definition of $\boldsymbol{d}_{i, t}$.

\section{Simulations}

In this section, we study the performance of the proposed algorithm under different scenarios. For the network structure, we consider a depth 2 tree-network. Each agent $i$ observes a noise corrupted version $y_{t} \in \mathbb{R}$ of an underlying state $x_{t} \in \mathbb{R}$, where it evolves according to the random walk model in (2) with $\gamma=0.98$. The state noise $w_{t}$ is driven by a Gaussian process, with zero mean and variance $\sigma_{w}^{2}=0.025$. The observation noise is also zero-mean white Gaussian random process.

We use the terminal cost function for measuring the team performance of different algorithms. The terminal cost is a function of the time horizon $T$ and defined as [29]

$J(T)=\sum_{i=1}^{m} \mathbb{E}\left\|\mathcal{X}_{T}-\hat{\chi}_{i, T}\right\|^{2}$,

which represents the impact of the final estimate on the horizon. We use ensemble average over 200 experiments in order to approximate the cost measure in (31).

We compare the performance of the proposed algorithm with the diffusion least mean squares (D-LMS), the diffusion recursive least squares (D-RLS) algorithm and the diffusion implementation of the Kalman filtering algorithm (D-Kalman) under different settings $[12,14,24]$. We also use a distributed consensus algorithm in our comparison framework [38]. In order to provide explanations for the implementations of these algorithms in our framework, we write a pseudocode in Algorithm 2. Note that since the implemen-

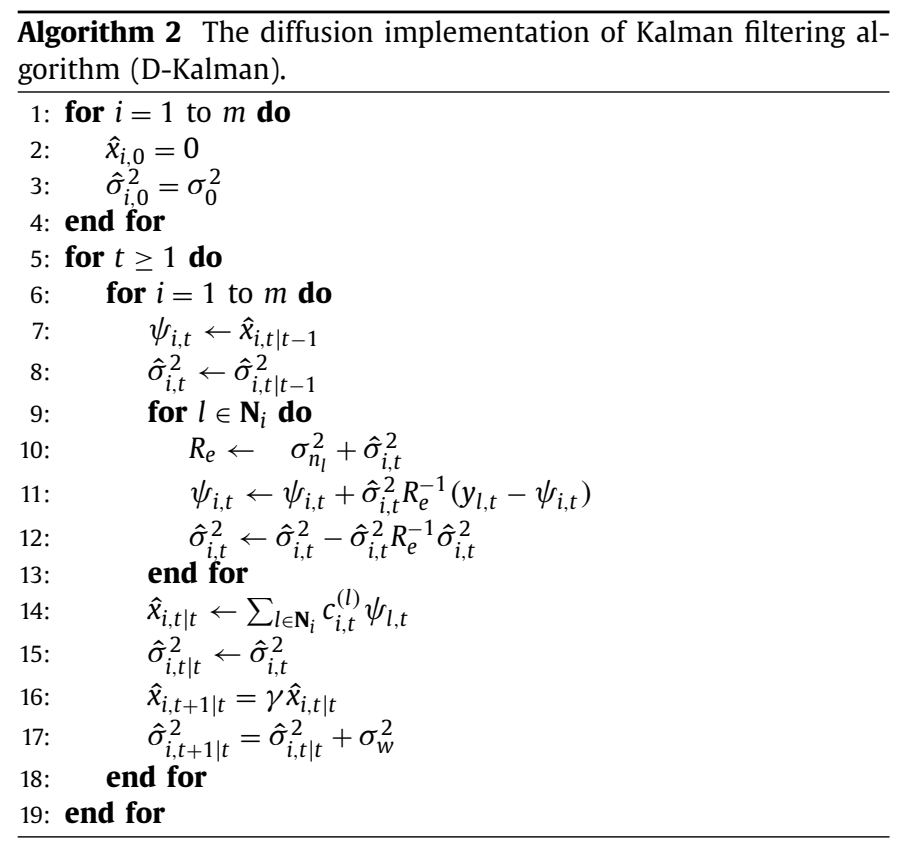

tation steps of these algorithms follow similar steps, we provide only one of them as an illustrative example in Algorithm 2.

We implement the diffusion based distributed algorithms with the adapt-then-combine (ATC) technique, where each agent first makes an estimate based on its local observation and discloses its estimate [38]. Then, the agents decide on their final estimate by combining the local and the received estimates. For the combination step, we use the Metropolis rule, where the combination weight $\lambda_{i, j}$ for the estimate coming to the agent $i$ from the agent $j$ 


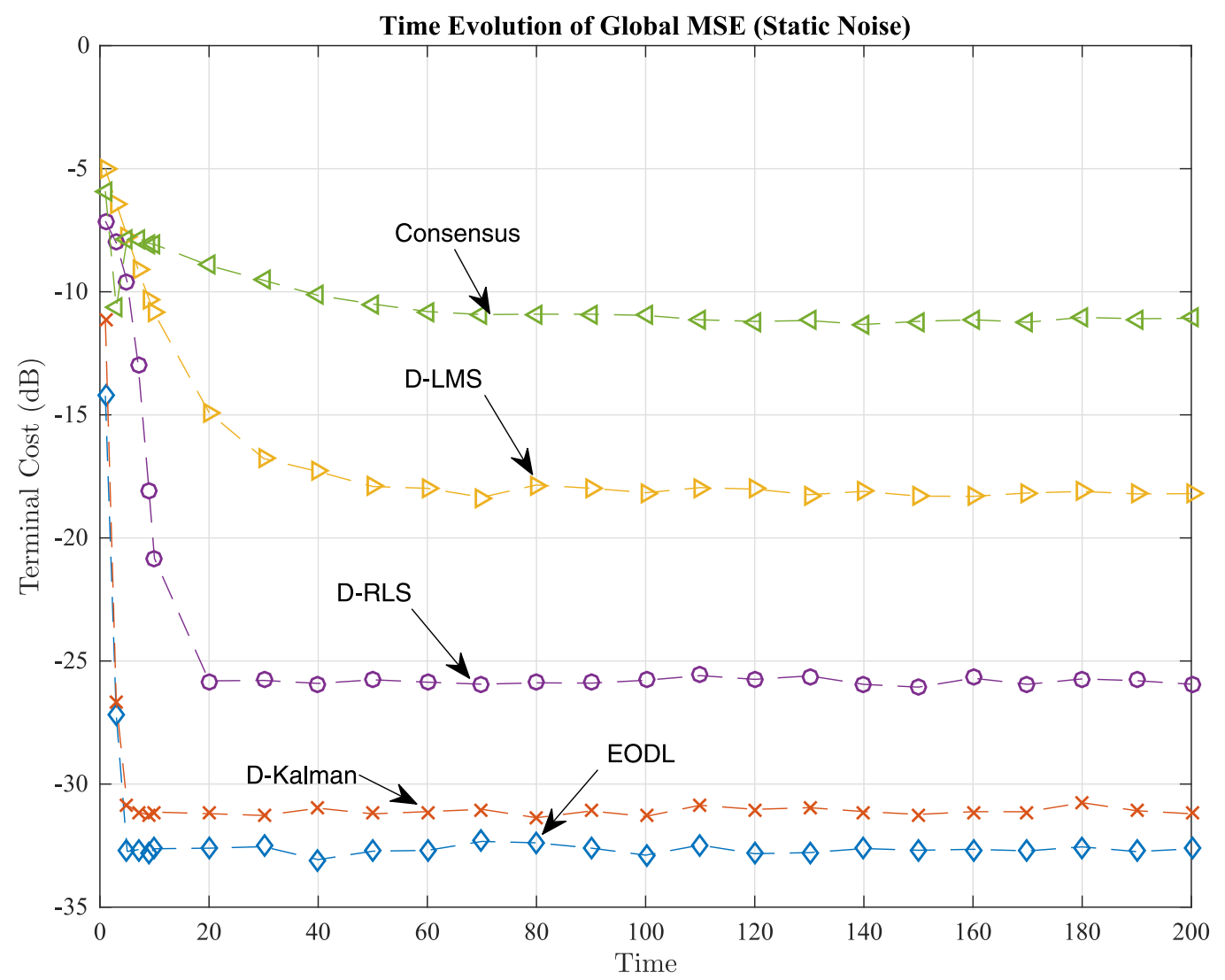

Fig. 4. Comparison of the global MSE of the algorithms under space-invariant noise with $\gamma=0.98$.

is calculated as

$\lambda_{i, j}= \begin{cases}\frac{1}{\max \left(\mathbf{N}_{i}, \mathbf{N}_{j}\right)} & \text { if } i \neq j \text { are linked, } \\ 0 & \text { for } i \text { and } j \text { not linked, } \\ 1-\sum_{j \in \mathcal{N}_{i} \backslash i} \lambda_{i, j} & \text { for } i=j .\end{cases}$

We set the learning rates of the diffusion LMS and the consensus algorithm to $\mu=0.2$. We select this learning rate so that these algorithms do not follow the observation noise and capture the underlying parameter. Also, we set the memory parameter of the diffusion RLS algorithm to $\eta=0.3$. Note that we set the memory parameter of RLS algorithm to a relatively small value in order to put more emphasis on the recent observations and estimates. We choose this parameter so that the diffusion RLS algorithm converges fast, but still be able to track the underlying dynamic parameter.

In Fig. 4, we compare the algorithms under a space-invariant noise over the network, where each agent experiences the same level of disturbance. We select the random walk and observation model parameter so that each agent experiences $0.5 \mathrm{~dB}$ signal-tonoise ratio (SNR). We observe that the proposed algorithm (EODL) achieves a superior performance regarding the global finite horizon MSE measure and the convergence rate compared to the other distributed estimation algorithms. The consensus algorithm performs the worst since it has a decaying learning rate as the nodes reach to a consensus in time and the network loses its adaptation capabilities against a dynamic parameter. In another scenario, we evaluate the performance of the algorithms under a spacevariant noise statistics over the network. For this case, we randomly sample the standard deviation of the observation noise of the each agent from a folded Gaussian distribution so that signalto-noise ratio of the network will be around $0.5 \mathrm{~dB}$. In this case randomness is involved and some of the agents will experience higher(lower) SNR levels. In Fig. 5, we compare the algorithms for the space-variant noise case. Note that the algorithms perform better than the space-invariant noise statistics case. This is because, in this case, some of the agents experience smaller noise levels while some others experience higher, but through the communication between the agents, they all benefit from the estimates of the agents having better observation channels. We also emphasize that the EODL algorithm even performs better in this case since it utilizes the optimal information disclosure and the estimate construction. Furthermore, we observe a similar performance between the compared algorithms, where the EODL algorithm achieves a superior performance regarding the global MSE measure and the convergence rate compared to the other distributed estimation schemes.

We also investigate the effect of the random walk parameter $\gamma$ with a simulation under the space-variant noise framework, thus we select $\gamma=1$ for another set of simulations. We emphasize that in this case the random walk model diverges, however, as we will observe, our algorithm provides a bounded estimation MSE. In Fig. 6, we present the results for the case of no cooperation between the nodes and for the case the cooperation occurs. We observe that the D-LMS, D-RLS and the consensus networks become unstable, even if individual nodes are stable and able to track the underlying parameter. Only the D-Kalman and the EODL networks are able to achieve the convergence for $\gamma=1$ case. We also emphasize that, with the EODL algorithm, we produce the optimal parameters and the combination weights for the network in contrast to the D-LMS, D-RLS and consensus algorithms, where we need to select their parameters beforehand. Therefore, the proposed algorithm overcomes the issue of parameter selection and provides more stable solution for this scenario. 


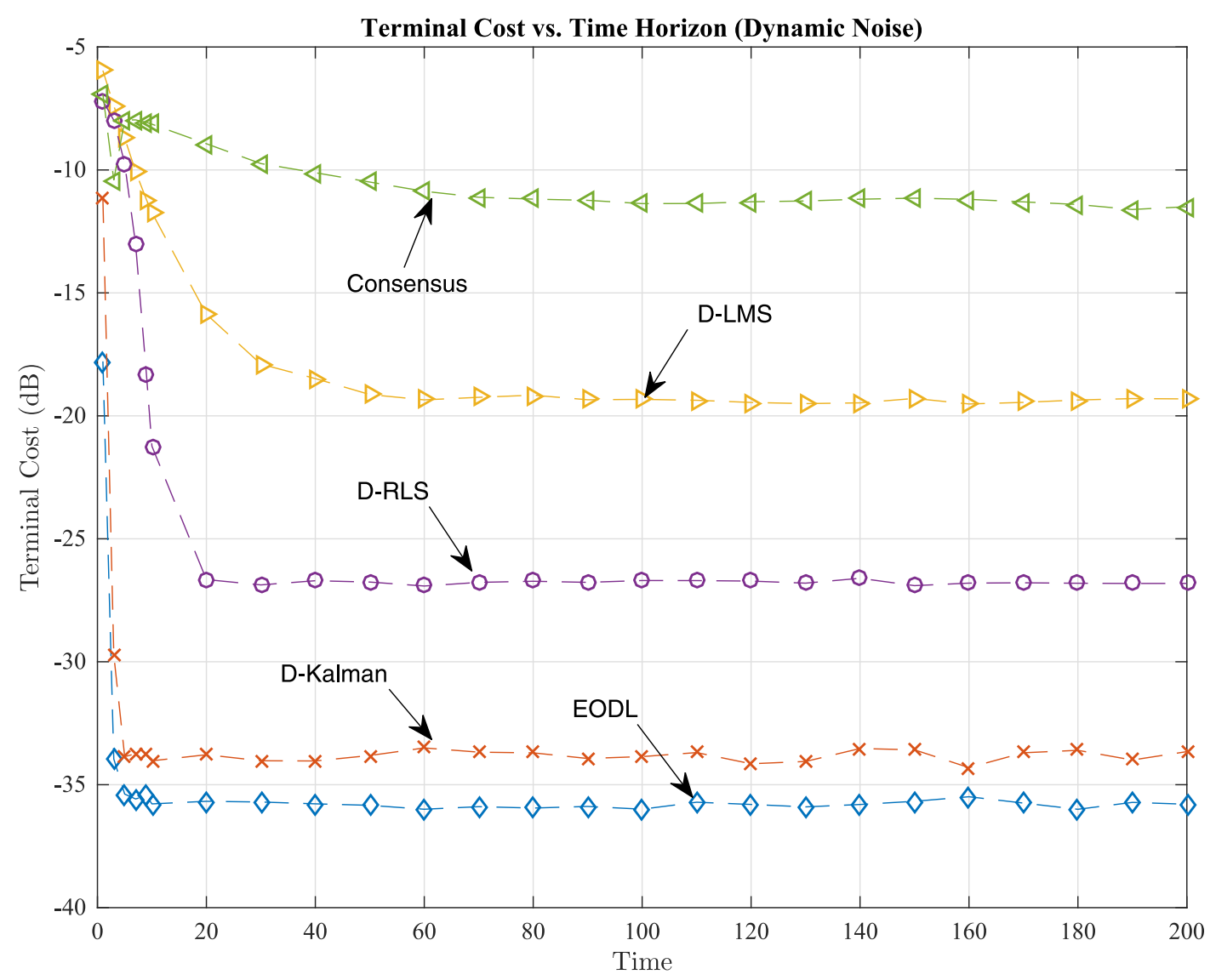

Fig. 5. Comparison of the global MSE of the algorithms under space-variant noise with $\gamma=0.98$.

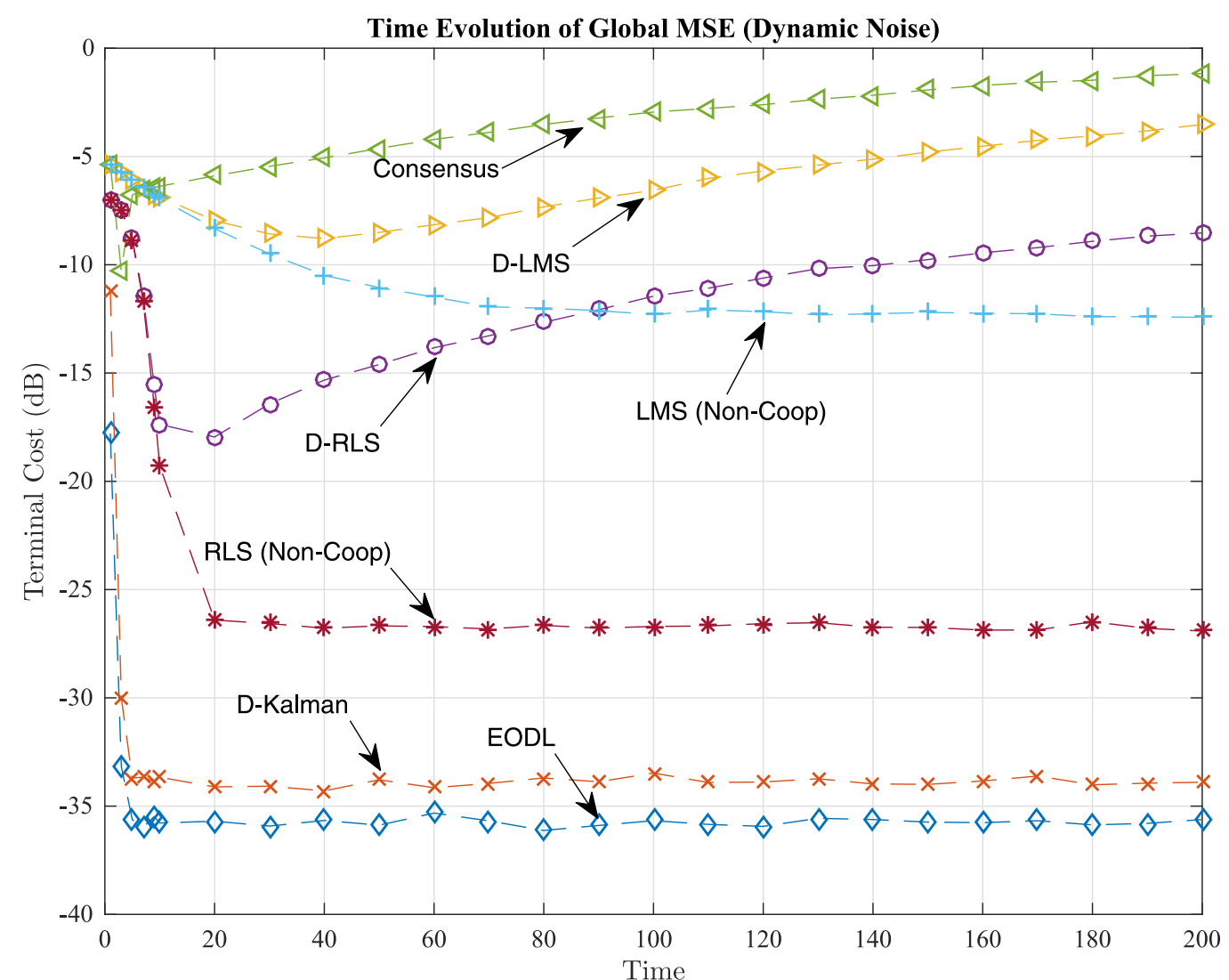

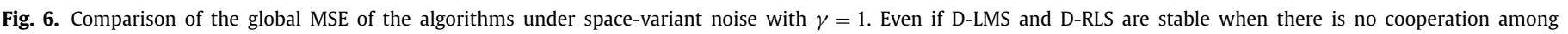
nodes, the networks are diverging when they cooperate. 


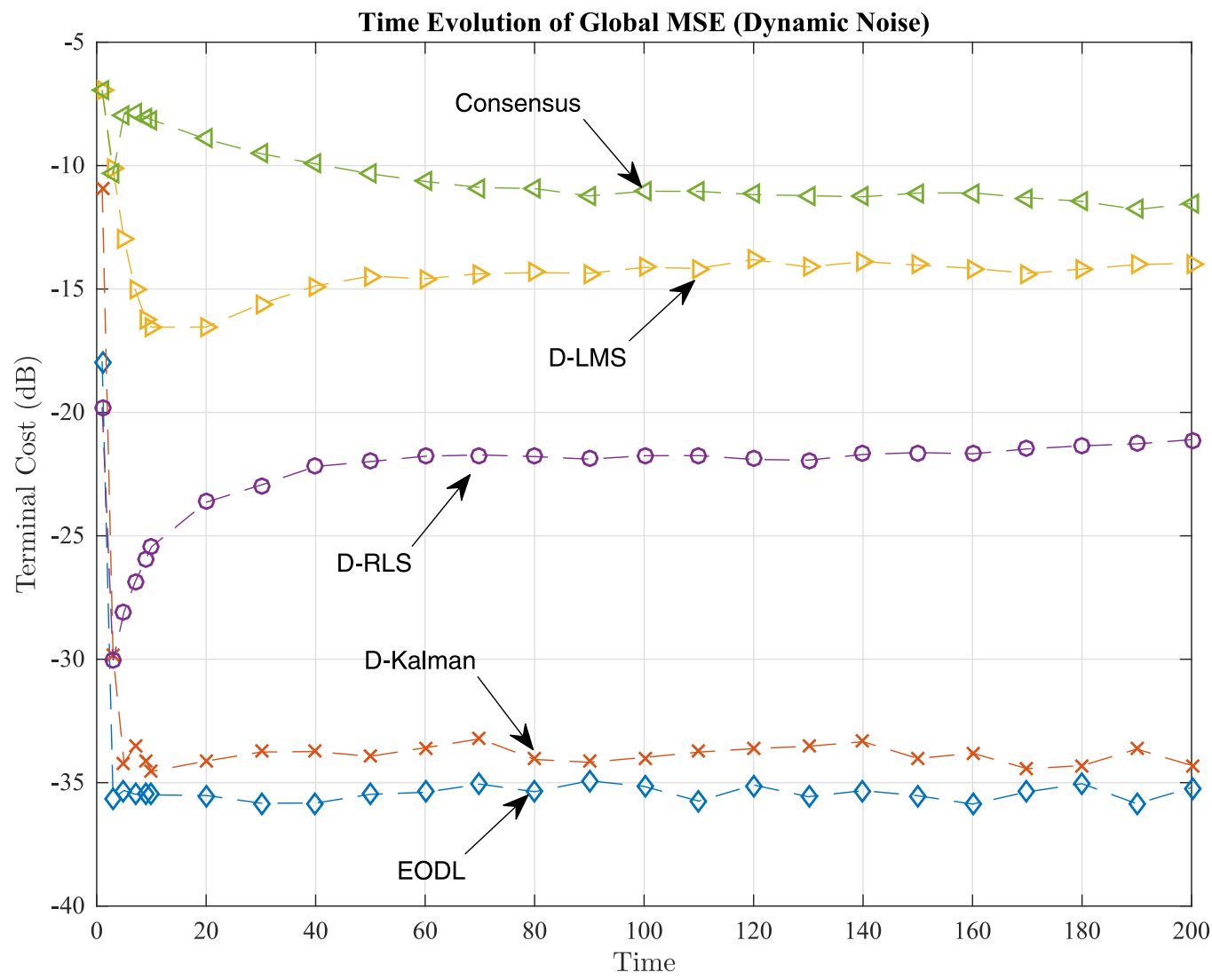

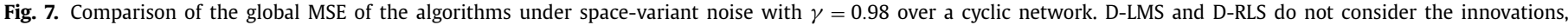
therefore they perform worse for the cyclic network case. The EODL algorithm shows the superior performance compared to the other algorithms.

Finally, in order to show the general applicability of the EODL algorithm, we consider a cyclic network. We set $\gamma=0.98$ and we use space variant noise statistics as in former case. In Fig. 7, we compare the performance of the algorithms for the cyclic network case. We note that even though the EODL algorithm does not perform the optimal estimation, through the recursive extraction of innovations and combination weights, it shows a superior performance compared to the other algorithms. We also emphasize that due to the structure of the network, the correlation among the disclosed information is increased and resulted in worse performance for the D-LMS and D-RLS algorithms compared to the tree-network case.

\section{Conclusion}

In this paper, we introduce a novel approach for the distributed estimation of dynamically changing parameters. We first construct a framework for the estimation of dynamic parameters by a team of distributed agents. Here, we provide a lower bound on the estimation error of the team of agents in the MSE sense. We prove that the lower bound can be achieved for any arbitrary network when the agents disclose the stamped observations. We also show that this method imposes huge communication loads and requires excessive storage on the agents. Therefore, we introduced an efficient method where the agents only disclose the "new information" they have collected. We prove that the error lower bound in this case can only be achieved over certain network topologies. We introduce an algorithm to recursively extract the innovations from the disclosed information and construct the optimal estimates. Through series of simulations over different scenarios, we illustrate the significant performance improvements introduced by our algorithm with respect to the state of the art methods.

\section{Acknowledgement}

This work is supported in part by. TUBITAK Contract No 117E153.

\section{References}

[1] A.H. Sayed, S.Y. Tu, J. Chen, X. Zhao, Z.J. Towfic, Diffusion strategies for adaptation and learning over networks: an examination of distributed strategies and network behavior, IEEE Signal Process. Mag. 30 (3) (2013) 155-171.

[2] A. Nedic, A. Ozdaglar, Distributed subgradient methods for multi-agent optimization, IEEE Trans. Automat. Contr. 54 (1) (2009) 48-61.

[3] S. Shahrampour, S. Rakhlin, A. Jadbabaie, Online learning of dynamic parameters in social networks, Advances in Neural Information Processing Systems, 2013.

[4] K. Tsianos, M.G. Rabbat, Efficient distributed online prediction and stochastic optimization with approximate distributed averaging, IEEE Trans. Signal Inf. Process. Networks 2 (4) (2016) 489-506.

[5] A. Dimakis, S. Kar, J.M.F. Moura, M.G. Rabbat, A. Scaglione, Gossip algorithms for distributed signal processing, Proc. IEEE 98 (11) (2010) 1847-1864.

[6] H. Salami, B. Ying, A.H. Sayed, Social learning over weakly connected graphs, IEEE Trans. Signal Inf. Process. Networks 3 (2) (2017) 222-238.

[7] L. Canzian, Y. Zhang, M. van der Schaar, Ensemble of distributed learners for online classification of dynamic data streams, IEEE Trans. Signal Inf. Process. Networks 1 (3) (2015) 180-194.

[8] D.D. Chaudhary, S.P. Nayse, L.M. Waghmare, Application of wireless sensor networks for greenhouse parameter control in precision agriculture, Int. J. Wireless Mobile Networks (IJWMN) 3 (1) (2011) 140-149.

[9] D. Estrin, L. Girod, G. Pottie, M. Srivastava, Instrumenting the world with wireless sensor networks, in: Acoustics, Speech, and Signal Processing, 2001. Proceedings.(ICASSP'01). 2001 IEEE International Conference on, 4, IEEE, 2001, pp. 2033-2036.

[10] C.G. Lopes, A.H. Sayed, Diffusion least-mean squares over adaptive networks: formulation and performance analysis, IEEE Trans. Signal Process. 56 (7) (2008) 3122-3136. 
[11] N. Takahashi, I. Yamada, A.H. Sayed, Diffusion least-mean squares with adaptive combiners: formulation and performance analysis, IEEE Trans. Signal Process. 58 (9) (2010) 4795-4810.

[12] F.S. Cattivelli, C.G. Lopes, A.H. Sayed, Diffusion recursive least-squares for distributed estimation over adaptive networks, IEEE Trans. Signal Process. 56 (5) (2008) 1865-1877.

[13] S.Y. Tu, A.H. Sayed, Diffusion strategies outperform consensus strategies for distributed estimation over adaptive networks, IEEE Trans. Signal Process. 60 (12) (2012) 6217-6234.

[14] F.S. Cattivelli, C.G. Lopes, A.H. Sayed, Diffusion strategies for distributed kalman filtering: formulation and performance analysis, Proc. Cognit. Inf. Process. (2008) 36-41.

[15] G. Soatti, M. Nicoli, S. Savazzi, U. Spagnolini, Consensus-based algorithms for distributed network-state estimation and localization, IEEE Trans. Signal Inf. Process. Networks 3 (2) (2017) 430-444.

[16] I.D. Schizas, G. Mateos, G.B. Giannakis, Distributed $\operatorname{lms}$ for consensus-based in-network adaptive processing, IEEE Trans. Signal Process. 57 (6) (2009) 2365-2382.

[17] V. Matta, P. Braca, S. Marano, A.H. Sayed, Distributed detection over adaptive networks: refined asymptotics and the role of connectivity, IEEE Trans. Signal Inf. Process. Networks 2 (4) (2016) 442-460.

[18] S. Joshi, S. Boyd, Sensor selection via convex optimization, IEEE Trans. Signal Process. 57 (2) (2009) 451-462.

[19] M. Shamaiah, S. Banerjee, H. Vikalo, Greedy sensor selection under channel uncertainty, IEEE Wireless Commun. Lett. 1 (4) (2012) 376-379.

[20] H. Durrant-Whyte, M. Stevens, E. Nettleton, Data fusion in decentralised sensing networks, in: 4th International Conference on Information Fusion, 2001, pp. 302-307.

[21] A.H. Sayed, S.Y. Tu, J. Chen, X. Zhao, Z.J. Towfic, Diffusion strategies for adaptation and learning over networks: an examination of distributed strategies and network behavior, IEEE Signal Process. Mag. 30 (3) (2013) 155-171.

[22] L. Xiao, S. Boyd, S. Lall, A scheme for robust distributed sensor fusion based on average consensus, in: Proceedings of the 4th international symposium on Information processing in sensor networks, IEEE Press, 2005, p. 9.

[23] J. Fernandez-Bes, L.A. Azpicueta-Ruiz, J. Arenas-García, M.T.M. Silva, Distributed estimation in diffusion networks using affine least-squares combiners, Digit Signal Process. 36 (2015) 1-14.
[24] F.S. Cattivelli, A.H. Sayed, Diffusion lms strategies for distributed estimation, IEEE Trans. Signal Process. 58 (3) (2010) 1035-1048.

[25] M.O. Sayin, N.D. Vanli, I. Delibalta, S.S. Kozat, Optimal and efficient distributed online learning for big data, in: Big Data (BigData Congress), 2015 IEEE International Congress on, IEEE, 2015, pp. 126-133.

[26] D. Acemoglu, A. Nedic, A. Ozdaglar, Convergence of rule-of-thumb learning rules in social networks, in: Decision and Control, 2008. CDC 2008. 47th IEEE Conference on, IEEE, 2008, pp. 1714-1720.

[27] R. Frongillo, G. Schoenebeck, O. Tamuz, Social learning in a changing world Internet Network Econ. (2011) 146-157.

[28] Z.J. Towfic, J. Chen, A.H. Sayed, On distributed online classification in the midst of concept drifts, Neurocomputing 112 (2013) 138-152, doi:10.1016/j.neucom. 2012.12.043.

[29] M.O. Sayin, S.S. Kozat, T. Bașar, Team-optimal distributed mmse estimation in general and tree networks, Digit Signal Process. 64 (2017) 83-95.

[30] A.H. Sayed, Fundamentals of Adaptive Filtering, John Wiley \& Sons, 2003.

[31] B.D.O. Anderson, J.B. Moore, Optimal filtering, Englewood Cliffs 21 (1979) 22-95.

[32] M.O. Sayin, S.S. Kozat, Compressive diffusion strategies over distributed networks for reduced communication load, IEEE Trans. Signal Process. 62 (20) (2014) 5308-5323.

[33] M.O. Sayin, S.S. Kozat, Single bit and reduced dimension diffusion strategies over distributed networks, IEEE Signal Process. Lett. 20 (10) (2013) 976-979.

[34] P. Humblet, A distributed algorithm for minimum weight directed spanning trees, IEEE Trans. Commun. 31 (6) (1983) 756-762.

[35] M. Khan, G. Pandurangan, V.S.A. Kumar, Distributed algorithms for constructing approximate minimum spanning trees in wireless sensor networks, IEEE Trans. Parallel Distrib. Syst. 20 (1) (2009) 124-139.

[36] D. Peleg, Distributed Computing: A locality-sensitive Approach, SIAM, 2000.

[37] M. Elkin, An unconditional lower bound on the time-approximation trade-off for the distributed minimum spanning tree problem, SIAM J. Comput. 36 (2) (2006) 433-456

[38] C.G. Lopes, A.H. Sayed, Diffusion least-mean squares over adaptive networks in: Acoustics, Speech and Signal Processing, 2007. ICASSP 2007. IEEE International Conference on, vol. 3, IEEE, 2007. III-917 